\title{
Doenças do sistema digestório de pequenos ruminantes no norte do Paraná*
}

\section{Digestive disorders of small ruminants in northern Paraná}

\author{
Gislaine Aparecida dos Santos Peruzi, ${ }^{* \star}$ Stefany Lia Oliveira Camilo, ${ }^{* \star *}$ Júlio Augusto Naylor Lisbôa, ${ }^{* \star \star *}$ Priscilla Fajardo Valente Pereira***
}

\begin{abstract}
Resumo
As doenças do sistema digestório de ovinos e caprinos no norte do Paraná foram avaliadas por meio de um estudo retrospectivo de 427 pequenos ruminantes atendidos no Ambulatório de Grandes Animais, do Hospital Veterinário da Universidade Estadual de Londrina, no período de janeiro de 2006 a dezembro de 2015. Para isso, foi realizada revisão das fichas clínicas dos pequenos ruminantes com afecções do sistema digestório, determinando a ocorrência, principais características clínicas, tratamentos instituídos e evolução dos casos. Os dados foram tabulados e submetidos a um estudo descritivo das variáveis, observando a distribuição das frequências (\%) das condições analisadas. As afeç̧ões do sistema digestório foram diagnosticadas em 38,64\% (165/427) dos atendimentos realizados. Vinte e seis animais apresentaram duas afecções no momento do atendimento, totalizando 191 enfermidades diagnosticadas. Dentre essas enfermidades, as mais comumente diagnosticadas foram: hemoncose $(27,7 \% ; 53 / 191)$, acidose láctica ruminal aguda $(18,8 \% ; 36 / 191)$, eimeriose $(13,6 \% ; 26 / 191)$ e indigestão simples $(6,8 \% ; 13 / 191)$. O aumento da criação de ovinos e caprinos no estado do Paraná reitera a importância do desenvolvimento de estudos como o presente trabalho, a fim de identificar as enfermidades mais frequentes e preparar o médico veterinário para o diagnóstico e tratamento correto.
\end{abstract}

Palavras-chave: afecções do sistema digestivo, casuística hospitalar, caprinos, ovinos.

\begin{abstract}
Sheep and Goat's digestive disorders in northern Paraná were evaluated by a retrospective study of 427 small ruminants treated at the State University of Londrina's Veterinary Hospital (HV-UEL), from January 2006 to December 2015. Analysis of medical records of small ruminants with digestive disorder were performed, determining the occurrence, main clinical signs, established treatments and outcome of cases. Data were tabulated and submitted to a descriptive study of variables, observing frequency distribution (\%) of analyzed conditions. Digestive disorders occurs in 38,6\% (165/427) of cases in small ruminants at the studied period. Among these diseases, the most common were: hemoncose $(27.7 \% ; 53 / 191)$, acute rumen lactic acidosis $(18.8 \%$; $36 / 191)$, eimeriosis $(13.6 \% ; 26 / 191)$ and simple indigestion $(6.8 \% ; 13 / 191)$. The increase in sheep and goats' flock in the state of Paraná reiterates the importance of developing studies such as the present study, in order to identify the most frequent diseases and prepare the veterinarian for the correct diagnosis and treatment.
\end{abstract}

Keywords: digestive disordes, casuistry, goat, sheep.

\section{Introdução}

A criação de ovinos e caprinos é uma atividade que vem se destacando dentre as criações, contribuindo para o desenvolvimento econômico e fornecimento de alimento à população. É uma prática realizada em diversas regiões do mundo, devido à capacidade de adaptação desses animais frente às mais variadas condições de solo e clima e, se realizada corretamente, pode contribuir para erradicação da pobreza de criadores em situação de risco (Polloti; Wilson, 2009; Ximenes e Cunha, 2012; Dubeuf et al., 2014). No Brasil, em 2018, o efetivo de pequenos ruminantes foi de cerca de 29 milhões de cabeças, sendo que o rebanho ovino foi relativamente maior que o caprino (18,9 milhões de ovinos e 10,7 milhões de caprinos), e $63,2 \%$ dos ovinos e $94 \%$ dos caprinos encontravam-se na região Nordeste. No estado do Paraná, o rebanho ovino foi de 556.512 cabeças enquanto o de caprinos foi 115.718 animais (Brasil, 2018).

Em 2006 foi criado o Programa de Apoio à Estruturação das Cadeias Produtivas de Caprinos e Ovinos do Paraná, conduzido pela Secretaria da Agricultura e do Abastecimento (SEAB), Instituto Agronômico do Paraná (IAPAR) e Instituto Paranaense de Assistência Técnica e Extensão Rural (EMATER-PR) que juntamente com o Projeto de Ovinos e Caprinos incentiva a criação de pequenos ruminantes no estado. O objetivo principal dos programas estaduais é desenvolver e viabilizar a ovinocultura e a caprinocultura como atividade de importância econômica e social, pela estruturação das cadeias produtivas por meio do associativismo. No estado do Paraná a ovinocultura destaca-se pela exploração de cordeiros para abate, os quais

*Recebido em 27 de abril de 2020 e aceito em 16 de junho de 2020.

**Médica Veterinária: Cativa - Cooperativa Agroindustrial de Londrina; Londrina, PR.

***Programa de Pós-Graduação; Universidade Estadual de Londrina; Centro de Ciências Agrárias; Departamento de Clínicas Veterinárias; Londrina, PR.

****Docente: Universidade Estadual de Londrina; Centro de Ciências Agrárias; Departamento de Clínicas Veterinárias; Londrina, PR. 
são oriundos de criações de pequeno e médio porte com planteis compostos por um número reduzido de matrizes e como atividade secundária à produção de outras espécies, principalmente a bovina. A caprinocultura do Paraná se destacou pela venda de reprodutores e evoluiu para a produção e carne e de leite e seus derivados, porém em escala menor que a ovinocultura (Paraná, 2003; Paraná, 2004).

As enfermidades do sistema digestório nos pequenos ruminantes são responsáveis por grandes perdas econômicas. A ocorrência desses distúrbios aumentou com a intensificação da produção, devido à alteração da dieta dos animais para serem obtidos índices produtivos superiores, por falhas no manejo, habitação de áreas de pastejo precárias e a superpopulação (Lira et al., 2013).

A literatura brasileira, em sua maioria, aborda os distúrbios digestórios de bovinos, por ser a parte majoritária do rebanho brasileiro, e as informações relacionadas aos pequenos ruminantes são escassas (Lira et al., 2013). Sendo assim, existe a tendência de se considerar que a etiologia e a patogenia dessas enfermidades sejam semelhantes nos pequenos ruminantes e bovinos. Mundialmente, os distúrbios digestórios relacionados aos pequenos ruminantes são as helmintoses gastrointestinais, doenças fermentativas dos pré-estômagos, coccidioses e clostridioses (Pugh et al., 2020; Smith; Van Metre; Pusterla, 2019; Constable et al., 2016). Poucos estudos sobre a prevalência de diferentes transtornos digestórios em pequenos ruminantes foram publicados no Brasil, principalmente oriundos da região Nordeste, e a maioria deles aborda enfermidades específicas (Lira et al., 2013), como para a para acidose ruminal e doenças parasitárias (Vieira et al., 2006; Silva et al., 2009; Costa; Simões; Riet-Correa, 2009).

Os estudos à respeito da ocorrência dessas emfermidades em pequenos ruminantes na região Sul, especialmente do estado do Paraná, são escassos. Portanto, este trabalho teve como objetivo relatar as principais enfermidades do sistema digestório de pequenos ruminantes, atendidos no Ambulatório de Grandes Animais do Hospital Veterinário da Universidade Estadual de Londrina, em um período de 10 anos (2006 - 2015).

\section{Material e métodos}

Foram analisadas às fichas clínicas dos pequenos ruminantes com afecções do sistema digestório, atendidos no Ambulatório de Grandes Animais do Hospital Veterinário da Universidade Estadual de Londrina no período de janeiro de 2006 a dezembro de 2015. Foram levantados dados das fichas clínicas referentes à anamnese como: idade, sexo, raça, evolução da doença, tratamentos realizados, manejo da propriedade e histórico de enfermidades anteriores. Foram detalhados dados referentes ao exame físico como frequência cardíaca, frequência respiratória, temperatura retal, movimentos ruminais, coloração das mucosas, grau de desidratação e anotações específicas sobre cada sistema; diagnóstico a partir da análise do exame clínico, associados à exames laboratoriais como hemograma, avaliação bioquímica sérica, urinálise e coproparasitológico, principalmente quantidade de ovos por grama de fezes (OPG); protocolo de tratamento e evolução dos casos. Sobre os animais que morreram por afecções do sistema digestório, informações foram obtidas junto ao laboratório de Patologia Animal.
Uma análise descritiva das variáveis estudadas foi realizada, observando a distribuição das frequências (\%) das condições clínicas registradas nos prontuários.

\section{Resultados e discussão}

Durante os dez anos pesquisados (2006 - 2015), foram atendidos 427 pequenos ruminantes, sendo registrados 38,6\% (165/427) atendimentos de animais com afecções do sistema digestório. Destes $62,4 \%$ (103/165) eram ovinos e 37,6\% caprinos (62/165), sendo $59,3 \%$ (98/165) fêmeas e 40,7\% (67/165) machos. Em relação a idade, $26 \%$ (43/165) dos animais possuíam idade inferior a 1 ano e $74 \%$ (122/165) animais com idade superior a 1 ano. Os animais atendidos eram 83,6\% (138/165) mestiços, sem raça definida. As raças predominantes em ovinos foram Santa Inês (10 animais) e Sulfolk (5 animais) e a raça Boer destacou-se nos caprinos (4 animais). A maioria dos pequenos ruminantes atendidos no HV-UEL eram criados em sistema semi-intensivo. Foram registradas $27,3 \%(45 / 165)$ mortes, das quais $80 \%$ (36/45) em ovinos e $20 \%$ (09/45) em caprinos. Foram diagnosticadas 28 enfermidades do sistema digestório (Tabela 1). Em $15,7 \%$ (26/165) dos animais duas enfermidades foram diagnosticadas concomitantemente, sendo 17 ovinos e nove caprinos. No total, 191 ocorrências de enfermidades do sistema digestório foram registradas.

A tabela 1 apresenta as enfermidades do sistema digestório diagnosticadas em pequenos ruminantes no período estudado. Dentre todas as enfermidades encontradas na pesquisa, hemoncose, acidose láctica ruminal aguda (ALRA), eimeriose e indigestão simples foram as mais frequentes, totalizando $67 \%$ $(128 / 191)$ das afecções. Outras afecções foram diagnosticadas em menor número.

Pode-se observar que a hemoncose foi a enfermidade mais prevalente dentre os ovinos (42/119), e nos caprinos representou a terceira enfermidade mais comum (11/72). Esse resultado se mostrou contrário aos estudos de Costa, Simões e RietCorreia (2009) e Lira et al. (2013), nos quais a hemoncose foi a enfermidade que mais acometeu os caprinos estudados, possivelmente devido à maior sensibilidade destes animais aos parasitas, segundo os autores. Acredita-se que o uso inadequado de vermífugos é um dos fatores de risco para maior ocorrência da hemoncose no Norte do Paraná. Os animais mais acometidos eram animais em fase de crescimento e fêmeas em lactação. No presente trabalho $60 \%(32 / 53)$ dos animais atendidos possuíam até 1 ano de idade e 30\% (16/53) eram fêmeas em idade reprodutiva. Dos animais atendidos no HVUEL, $16(30,2 \%)$ morreram. No estudo de Lira et al. (2013), as helmintíases gastrintestinais levaram à morte de $33,1 \%$ dos caprinos e ovinos atendidos, sendo considerada uma alta taxa de mortalidade, semelhante ao resultado do presente estudo.

Dos 53 animais acometidos por hemoncose, foi possível a revisão das fichas de 41 deles, em que se observou como principais formas de tratamento, a administração de antiparasitários, fluidoterapia, transfusão sanguínea, ingestão forçada de forragem verde e estimulantes do metabolismo celular e eritropoiese (Tabela 2).

O tratamento antiparasitário foi instituído em 36 animais (87,8\%). Em 27 deles, o antiparasitário foi administrado uma única vez; em seis, duas vezes; e em três animais ocorreram três ou mais 
Tabela 1: Afecções e procedimentos do sistema digestório de ovinos e caprinos diagnosticadas no Hospital Veterinário da Universidade Estadual de Londrina, Londrina, PR, no período de janeiro de 2006 a dezembro de 2015

\begin{tabular}{|c|c|c|c|c|c|c|c|}
\hline \multirow{2}{*}{$\begin{array}{l}\text { Enfermidades e procedimentos } \\
\text { realizados }\end{array}$} & \multirow{2}{*}{$\begin{array}{l}N^{\circ} \text { de } \\
\text { casos }\end{array}$} & \multicolumn{2}{|c|}{ Ovinos } & \multicolumn{2}{|c|}{ Caprinos } & \multicolumn{2}{|c|}{ Letalidade } \\
\hline & & $\mathrm{N}$ & $\%$ & $\mathrm{n}$ & $\%$ & $\mathrm{n}$ & $\%$ \\
\hline Hemoncose & 53 & 42 & 77,4 & 11 & 22,6 & 16 & 30,2 \\
\hline Acidose láctica ruminal aguda & 36 & 15 & 41,7 & 21 & 58,3 & 8 & 21,6 \\
\hline Eimeriose & 26 & 11 & 42,3 & 15 & 57,7 & 9 & 34,6 \\
\hline Indigestão simples & 13 & 10 & 76,9 & 3 & 23,1 & 1 & 7,7 \\
\hline Deficiência proteica e mineral crônica & 12 & 5 & 41,7 & 7 & 58,3 & 6 & 50 \\
\hline Ruminostomia eletiva & 11 & 11 & 100 & 0 & 0 & 1 & 9,1 \\
\hline Diarreia (sem identificação do agente) & 7 & 4 & 57,1 & 3 & 42,9 & 3 & 42,9 \\
\hline Verminose por estrongilídeos & 6 & 2 & 33,3 & 4 & 66,7 & 0 & 0 \\
\hline Pancreatite (secundária a euritrematose) & 3 & 3 & 100 & 0 & 0 & 3 & 100 \\
\hline Prolapso retal & 3 & 2 & 66,7 & 1 & 33,3 & 0 & 0 \\
\hline Giardíase & 2 & 0 & 0 & 2 & 100 & 2 & 100 \\
\hline Histofilose & 2 & 2 & 100 & 0 & 0 & 2 & 100 \\
\hline Fotossensibilização hepática & 2 & 1 & 50 & 1 & 50 & 1 & 50 \\
\hline Megaesôfago funcional & 1 & 1 & 100 & 0 & 0 & 0 & 0 \\
\hline Enteroxemia por Clostridium spp. & 1 & 1 & 100 & 0 & 0 & 1 & 100 \\
\hline Monieziose & 1 & 1 & 100 & 0 & 0 & 1 & 100 \\
\hline Regurgitação a esclarecer & 1 & 1 & 100 & 0 & 0 & 0 & 0 \\
\hline Sialorréia a esclarecer & 1 & 1 & 100 & 0 & 0 & 0 & 0 \\
\hline Síndrome do bebedor ruminal & 1 & 0 & 0 & 1 & 100 & 0 & 0 \\
\hline Insuficiência hepática & 1 & 1 & 100 & 0 & 0 & 1 & 100 \\
\hline Obstrução intestinal & 1 & 1 & 100 & 0 & 0 & 1 & 100 \\
\hline Esofagite traumática & 1 & 1 & 100 & 0 & 0 & 0 & 0 \\
\hline Salmonelose & 1 & 1 & 100 & 0 & 0 & 1 & 100 \\
\hline Atresia anal & 1 & 0 & 0 & 1 & 100 & 0 & 0 \\
\hline Úlcera de abomaso & 1 & 0 & 0 & 1 & 100 & 0 & 0 \\
\hline Peritonite & 1 & 1 & 100 & 0 & 0 & 0 & 0 \\
\hline Periostite alveolar & 1 & 1 & 100 & 0 & 0 & 0 & 0 \\
\hline TOTAL & 191 & 119 & - & 72 & - & 57 & - \\
\hline
\end{tabular}

aplicações. Outros cinco animais vieram a óbito antes mesmo de se instituir o tratamento. Os princípios ativos mais utilizados foram Levamisol (7,5mg/kg, via subcutânea, (SC)) e Moxidectina $(0,2 \mathrm{mg} / \mathrm{kg}$, via subcutânea (SC)). É recomendado que a atuação do antiparasitário seja avaliada por exames coproparasitológicos realizados pré utilização e de 7 a 10 dias após, para estimativa da resistência antihelmíntica (Smith; Van Metre; Pusterla, 2019).

Em 11 animais, o tratamento foi diferenciado, pois esses apresentavam quadro clínico de diarreia e foram diagnosticados concomitantemente com eimeriose com base nos resultados do exame coproparasitológico. A utilização de antimicrobiano aconteceu em sete dos 11 animais, e como os mais utilizados observou-se a Oxitetraciclina $(20 \mathrm{mg} / \mathrm{kg}$, via intramuscular (IM), a cada 48 horas) e Sulfametoxazol e Trimetoprima ( $25 \mathrm{mg} / \mathrm{kg}$, IM). Nos outros quatro animais acometidos concomitantemente com eimeriose, o Toltrazuril $(20 \mathrm{mg} / \mathrm{kg}, \mathrm{VO})$, dose única), foi o coccidiostático eleito para o tratamento.

A administração de fluidoterapia foi realizada em oito animais e a transfusão sanguínea em sete animais acometidos, esta última com a finalidade de minimizar a anemia grave instalada. O volume de sangue transfundido variou dependendo do peso do animal, hematócrito do receptor e hematócrito pretendido, segundo a fórmula de Hunt e Moore (1990). A ingestão forçada só foi listada em três animais que apresentavam anorexia. Suplemento vitamínico foi utilizado em nove animais com o intuito de repor nutrientes (Tabela 2). 
Tabela 2: Tratamentos instituídos aos pequenos ruminantes diagnosticados com hemoncose no Hospital Veterinário da Universidade Estadual de Londrina, Londrina/PR, no período de janeiro de 2006 a dezembro de 2015

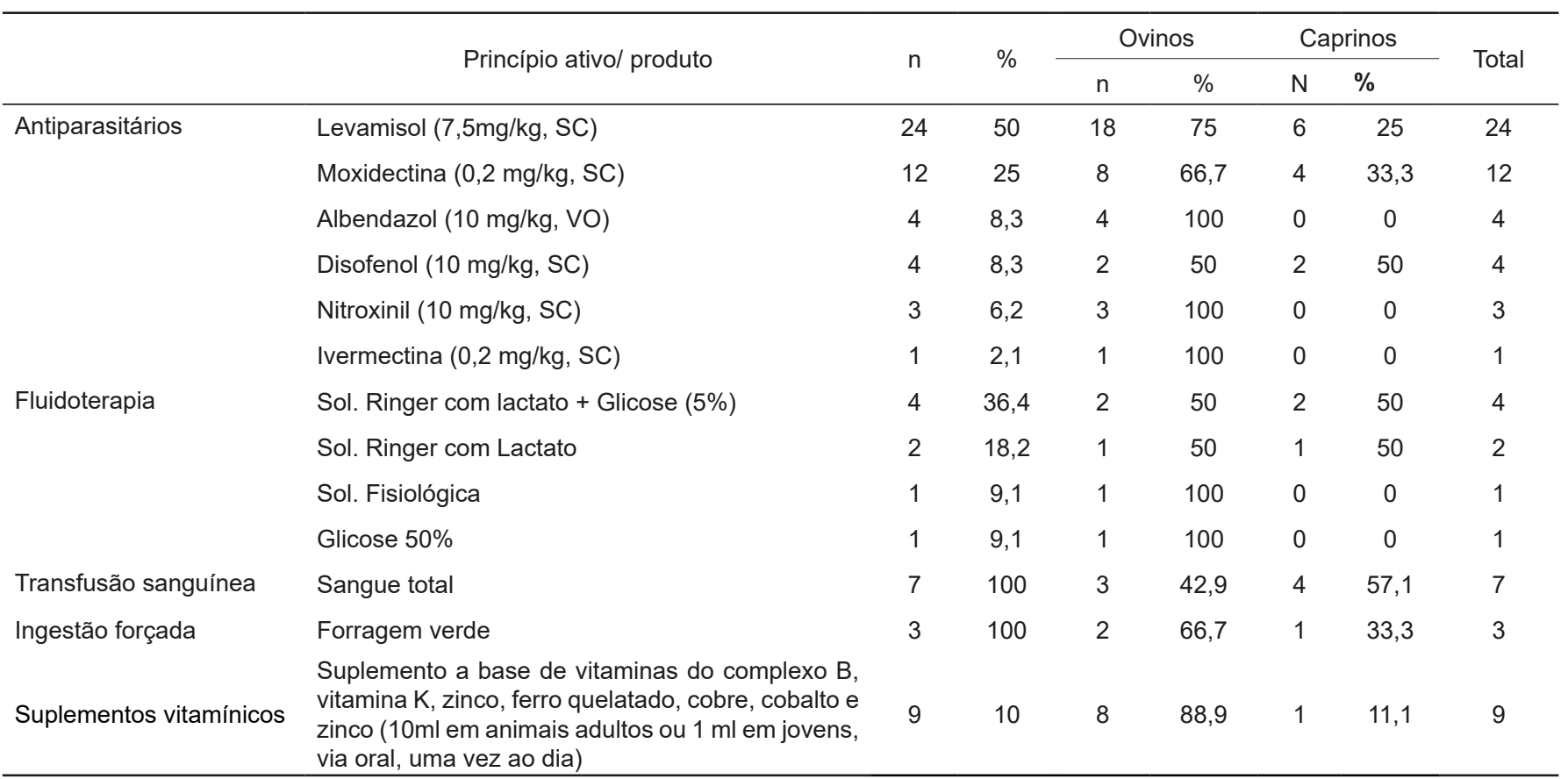

Dos 36 casos de ALRA diagnosticados, 21 (58,3\%) ocorreram em caprinos e $15(41,7 \%)$ em ovinos. O maior número de caprinos afetados pela enfermidade se justifica pelos animais atendidos serem criados no sistema semi-intensivo, e alimentados com grande quantidade de concentrado para produção de carne e/ou leite, segundo histórico das fichas clínicas. Na maioria das vezes, os animais não passaram por uma adaptação para este tipo de alimentação, e em outros a utilização prévia destes alimentos ocorreu por longo período. Quinze dos 21 caprinos acometidos eram de uma mesma propriedade e a doença aconteceu em surto, resultando na morte de três animais. Todos os animais diagnosticados apresentavam $\mathrm{pH}$ do suco ruminal abaixo de 5 , confirmando o diagnóstico de ALRA.

Dos 36 animais investigados, foi possível a revisão da ficha clínica de 32, sendo que 19 deles apresentaram ALRA leve e/ou em fase de resolução, sendo recomendada alimentação adequada com alimento verde ou feno. Os 13 animais restantes apresentaram acidose láctica ruminal moderada a grave, classificada de acordo com os resultados de gasometria ( $\mathrm{pH}$ e excesso de bases (BE)) e intensidade dos sinais clínicos (Constable et al., 2016). Em relação aos procedimentos terapêuticos utilizados nestes casos, em oito animais foi realizada lavagem ruminal por sonda ororuminal, utilizando em média 12 litros de água morna para retirada do conteúdo acidificado do rúmen. Este procedimento também foi utilizado nos estudos de Vieira et al. (2006), Lira et al. (2013) e Pereira et al. (2018). A transfaunação foi realizada em cinco animais uma única vez, e em três animais repetidas vezes. Esta prática é citada em diversos estudos sobre ALRA e, não há protocolo estabelecido sobre o volume de líquido ruminal a ser transferido. Pode ser realizada administração única (Lira et al., 2013; Vieira et al., 2006, Pereira et al., 2018) ou repetida (Braum et al., 1992), com volume de um a dois litros recomendado em pequenos ruminantes adultos. Dez animais receberam fluidoterapia intravenosa composta por solução de ringer com lactato e solução de bicarbonato de sódio a 1,3\%, e um animal recebeu fluidoterapia via oral (solução à base de $\mathrm{NaCl}, \mathrm{KCl}$, acetato de sódio trihidratado e D-glicose anidra (Bregadioli et al., 2018)) e solução de bicarbonato de sódio a 6\%, por via intravenosa. Frequentemente, o tratamento clínico com a adição de bicarbonato de sódio é realizado em animais com casos graves da doença (Braum et al., 1992; Vieira et al., 2006).

A ocorrência da Eimeriose foi frequente nos animais atendidos no HV-UEL durante o período pesquisado, com maior ocorrência nos caprinos $(57,7 \%)$, do que nos ovinos $(42,3 \%)$. No estudo de Lira et al. (2013), a eimeriose foi diagnosticada principalmente em caprinos jovens, o que está de acordo com o presente trabalho em que $50 \%$ (13/26) dos animais acometidos possuíam até 90 dias de idade. Os animais jovens, com média de idade de 60 dias de vida, são mais susceptíveis, devido à ausência de imunidade desta categoria frente à infecção (Costa et al., 2009).

$\mathrm{Na}$ indigestão simples, a prevalência em ovinos foi mais alta que em caprinos. Nesta enfermidade o que se destaca é a elevada porcentagem de alta hospitalar $(92,3 \%)$ em relação às mortes $(7,7 \%)$. Esse fato ocorre por se tratar de uma doença com sintomatologia branda, quando comparada à ALRA (Smith; Van Metre; Pusterla, 2019). Segundo Lira et al. (2013), quadros de indigestão simples geralmente não necessitam de tratamento e são autolimitantes. No entanto, realizar procedimentos como fluidoterapia oral (solução à base de $\mathrm{NaCl}, \mathrm{KCl}$, acetato de sódio trihidratado e D-glicose anidra (Bregadioli et al., 2018)) e transfaunação poderiam diminuir o tempo de evolução da enfermidade.

É importante ressaltar que os casos identificados como verminose por estrongilídeos, são considerados aqueles em que o exame coproparasitológicos revelou a presença dos parasitas, 
porém, como não foi realizada cultura de fezes para identificação do tipo de estrongilídeo presente, estes foram categorizados conjuntamente.

Já os casos identificados como histofilose e insuficiência hepática foram diagnosticados post-morten, por meio do exame histopatológico.

Dentre as enfermidades do sistema digestório de menor ocorrência que merecem destaque por peculiaridades diagnósticas ou terapêuticas encontram-se: pancreatite por parasitismo de Eurytrema sp. em três animais, giardíase em dois animais, monieziose, úlcera de abomaso e síndrome do bebedor rumenal com uma ocorrência cada.

A pancreatite foi identificada em três ovinos atendidos, de uma mesma propriedade, sendo um no ano de 2012, e os outros dois no ano de 2013. Os animais apresentavam-se apáticos, caquéticos, com edema submandibular, dois apresentavam salivação esverdeada e um apresentava desconforto abdominal. Aos exames complementares, foram observados aumento da enzima gamaglutamiltransferase $(81 \mathrm{u} / \mathrm{L})$ em um dos casos e hiperglicemia $(263 \pm 13 \mathrm{mg} / \mathrm{dL})$ nos três animais. Os demais parâmetros se encontravam no intervalo fisiológico para a espécie e o exame coproparasitológico foi negativo. Estes animais foram submetidos à eutanásia, e em seguida necropsiados, que revelou alterações compatíveis com granulomas parasitários em pâncreas, compatíveis com euritrematose. Esta é uma enfermidade comum em bovinos e endêmica em diversos estados, incluindo o Paraná. Na maioria das vezes ocorre de forma subclínica, mas pode apresentar-se clinicamente como caquexia, anorexia, salivação espumosa e hiperglicemia (Azevedo et al., 2004; Bassani et al., 2007), já diagnosticada em ovinos no Rio Grande do Sul (Costa et al., 1986). Nos bovinos, a pancreatite é diagnosticada na maioria das vezes no abate, com detecção de nódulos parasitários em pâncreas, característicos da doença (Bassani et al., 2007), assim como os encontrados nos animais do presente trabalho.

A giardíase é uma zoonose comumente relatada em ruminantes e é considerada uma causa importante de diarreia em seres humanos e animais. É transmitida via oro-fecal por meio de água e alimentos contaminados com o parasita. Estudos brasileiros relatam que a prevalência em caprinos varia entre $22,6 \%$ e $29,3 \%$ e em ovinos entre $24 \%$ e $34 \%$ (Coelho et al., 2017; Sudré et al., 2014). No entanto, em estudo recente realizado em Botucatu (São Paulo) a prevalência de cistos de Giardia sp. foi de apenas 6,6\% (Moraes et al., 2019). Essa variação justificase pelas diferenças entre os trabalhos, relacionadas à idade dos animais, tipo de tipo de criação, técnicas de diagnóstico empregadas, condição sanitária das instalações e acesso de animais de companhia às instalações. Os dois casos de giardíase referidos no presente estudo ocorreram em caprinos, fêmeas de 90 dias, concomitantemente a casos de eimeriose. O diagnóstico foi definido com base nos achados do exame coproparasitológicos.

A monieziose é considerada de baixa patogenicidade em animais adulto e sua ocorrência é eventual (Pugh et al., 2020). O caso relatado neste trabalho ocorreu em um ovino de dois meses de idade, mantido a pasto e alimentado com silagem de milho, proveniente de um rebanho em que a vermifugação é realizada quinzenalmente com o auxílio do método de FAMACHA ${ }^{\circledR}$. O ovino apresentava-se apático, com $10 \%$ de desidratação, mucosas pálidas e diarreia, os exames laboratoriais indicavam hipoproteinemia $(3,2 \mathrm{~g} / \mathrm{dL})$ e presença de estrongilídeos (5050 opg), Eimeria sp (+). e Moniezia sp. O animal morreu e na necropsia foi observada parasitose intestinal acentuada, principalmente em intestino delgado, onde encontrou-se grande quantidade do cestódeo em toda a sua extensão, sendo o diagnóstico comclusivo.

A úlcera de abomaso é uma enfermidade pouco frequente em ovinos e caprinos, porém possui alto risco de morte (Smith; Van Metre; Pusterla, 2019). O caprino atendido foi internado uma primeira vez com queixa de fezes líquidas, e em seus exames complementares foi encontrada azotemia pré renal (ureia: $147 \mathrm{mg} / \mathrm{dL}$; creatinina: $9,0 \mathrm{mg} / \mathrm{dL}$ ), provavelmente devido à desidratação. Depois de tratado com uma aplicação de Moxidectina $(0,5 \mathrm{mg} / \mathrm{kg})$, Florfenicol por 3 dias $(20 \mathrm{mg} / \mathrm{kg})$ e fluidoterapia oral (solução à base de $\mathrm{NaCl}, \mathrm{KCl}$, acetato de sódio trihidratado e D-glicose anidra (Bregadioli et al., 2018)), o proprietário retirou animal para continuidade do tratamento em casa. Sete dias depois, o mesmo voltou ao hospital com fezes escurecidas e amolecidas, além de apresentar bruxismo. A azotemia pré renal persistia (Ureia: $76 \mathrm{mg} / \mathrm{dL}$; creatinina: 3 $\mathrm{mg} / \mathrm{dL}$ ) e adicionalmente, detectou-se acidose metabólica leve (Excesso de base: -3,9 mmol/L). Suspeitou-se, então, de úlcera de abomaso. O tratamento consistiu em hidratação intravenosa com Ringer com lactato, omeprazol por via intravenosa $(0,4 \mathrm{mg} /$ $\mathrm{kg})$ por 7 dias, aplicação única de metroclopramida $(0,2 \mathrm{mg} /$ $\mathrm{kg}, \mathrm{IV}$ ) e ingestão forçada de volumoso até retorno do apetite. Ao longo do tratamento, as fezes adquiriram consistência e coloração normal da espécie e o animal teve alta dentro de sete dias.

A síndrome do bebedor rumenal é uma afecção de ruminantes lactentes, que ocorre por não fechamento do sulco reticular ou por meio de refluxo abomasal, acarretando em retenção prolongada ou repetida do leite nos pré-estômagos, o que pode levar ao desenvolvimento de padrões de fermentação anormais (Silva et al., 2016). O animal atendido era um caprino, macho, de dois meses, mantido em piquete com a mãe e alimentando-se de leite materno. Seus sinais clínicos consistiam em sialorréia, bruxismo, apatia, rúmen dilatado por gás e líquido e fezes pastosas de coloração acinzentada. Foi realizada sondagem ruminal com drenagem de leite fermentado ( $\mathrm{pH} 5)$, indicando possível acúmulo reticuloruminal de leite. $\mathrm{O}$ tratamento instítuido consistiu de Ranitidina $(15 \mathrm{mg} / \mathrm{kg}$, IV) por seis dias, Sulfametoxazol e Trimetropima $(25 \mathrm{mg} / \mathrm{kg}, \mathrm{IM})$ por cinco dias e mudança gradativa na alimentação para forrageiras, progredindo para a alta hospitalar. Segundo a literatura, o tratamento preconizado é sintomático e consiste em: desmame, com troca para alimentação à base de volumoso; correção do $\mathrm{pH}$ ruminal alterado; e antibioticoterapia instituída baseada nos achados clínicos e hematológicos. As medidas terapêuticas têm a finalidade de auxiliar os movimentos ruminais, o reflexo de eructação e as ruminações a retornarem ao normal, o que acontece em uma a duas semanas (Garry, 2009; Smith; Van Metre; Pusterla, 2019).

\section{Conclusão}

As afecções do sistema digestório nos ovinos e caprinos representam parcela considerável das enfermidades destes animais no norte do Paraná. As enfermidades mais frequentes 
foram hemoncose, acidose láctica ruminal aguda, eimeriose e indigestão simples. Enfermidades que foram diagnosticadas em menor número devem ser consideradas como diagnósticos diferenciais para enfermidades do sistema digestório. As informações espécificas sobre enfermidades de pequenos

\section{Referências}

AZEVEDO, J.R.; MANNIGEL, R.C.; AGULHON, A.Z.; BORBA, T.R.; BARBIERI, A.W.; OLIVEIRA, D.C.L.; HEADLEY, S.A.; JANEIRO, V. Prevalence and geographic distribution of bovine eurytrematosis in cattle in northern Paraná, Brazil. Pesquisa Veterinária Brasileira, v.21, n.1, p.23-26, 2004.

BASSANI, C.A; SANGIONI, L.A; SAUT, J.P.E; HEADLEY, S.A; YAMAMURA, M.H. Euritrematose bovina. Semina: Ciências Agrárias, v.28, n.2, p.299-316, abr/jun 2007.

BRAUN, U; RIHS, T; SCHEFER, U. Ruminal lactic acidosis in sheep and goats. The Veterinary Record, v.130, p.343-349, abr 1992.

BRASIL. Instituto Brasileiro de Geografia e Estatística: IBGE. Produção da Pecuária Municipal 2018. Brasília, 2018. Disponivel em: < https://sidra.ibge.gov.br/tabela/3939> Acesso em: 20 de abril de 2020 .

BREGADIOLI, G.C.; PINTO, F.C; CURTI, J.M.; CAMILO, S.L.O.; FLAIBAN, K.K.M.C.; LISBÔA, J.A.N. Impacto de diferentes soluções eletrolíticas orais sobre a glicemia e os equilíbrios hídrico, eletrolítico e ácido-base de bezerros neonatos sadios Arquivo Brasileiro de Medicina Veterinária e Zootecnia, v.70, n.5, p.1433-1442, 2018.

COELHO, C.H.; DURIGAN M.; LEAL D.A.G.; SCHNEIDER A.B.; FRANCO R.M.B; SINGER S.M. Giardiasis as a neglected disease in Brazil: Systematic review of 20 years of publications. PLOS Neglected Tropical Disease, v.11, n.10, 2017.

COSTA, H.M.A; LEITE, A.C.R; GUIMARÃES, M.P; LIMA, W.S. Distribuição de helmintos parasitas de animais domésticos no Brasil. Arquivo Brasileiro de Medicina Veterinária e Zootecnia, v.38, n.4, p.465-579, 1986.

COSTA, V.M.M; SIMÕES, S.V.D; RIET-CORREIA, F. Doenças parasitárias em ruminantes no semi-árido brasileiro. Pesquisa Veterinária Brasileira. v.29, n.7, p. 563-568, jul 2009.

CONSTABLE, P.D.; HINCHCLIFF, K.W; DONE, S; GRUENBERG, W. Veterinary Medicine. A textbook of the diseases of cattle, horses, sheep, pigs, and goats. 11 ed. Filadélfia: Saunders Ltd, 2016. 2v. 2278p.

DUBEUF, J.; BENDAPUDI, R.; Bhandari, D.; CAPOTE, J.; CARRASCO-SANCHEZ, R.; DASKIRAN, I.; GUIMARAES, V.; INIGUEZ, L.; KOLUMAN-DARCAN, N.; PEACOCK, C.; ROTA, A.; RICHKOWSKY, B.; SEPE, L. Scaling up successful practices for pro-poor development projects involving goats: First outputs of a comparative study. Small Ruminant Research, v.121, n.1, p.146156, 2014.

GARRY, F.B. Rumen Indigestion and Putrefaction. In: ANDERSON, D.E; RINGS, D.M. Current Veterinary Therapy: Food Animal Practice. 5.ed. St Louis: Saunders Elsevier, 2009. p. 20-30.

LIRA, M.A.A; SIMÕES, S.V.D; RIET-CORREA, F; PESSOA, C.M.R; DANTAS, A.F.M; MIRANDA NETO, E.G. Doenças do sistema digestório de caprinos e ovinos no semiárido do Brasil. Pesquisa Veterinária Brasileira. v.33, n.2, p.193-198, fev 2013. ruminantes ainda são escassas, e por suas particularidades nem sempre devem ser comparados aos bovinos. Por isso, estudos como o desenvolvido são extremamente relevantes, uma vez que a criação de ovinos e caprinos tem crescido consideravelmente na região norte do Paraná.

MORAES, L.F.; KOZLOWSKI, N.; ANTONIO, V.; OLIVEIRA, R.M.; PROVIDELO, G.A.; BABBONI, S.D.; FERREIRA, J.C.P.; SCHMIDT, E.M.S. Estudo retrospectivo e comparativo da prevalência de Giardia sp. em cães, gatos e pequenos ruminantes em áreas endêmicas em diferentes estados brasileiros / Retrospective and comparative study of Giardia sp. prevalence in dogs, cats, and small ruminants in endemic areas in different Brazilian states. Acta Scientiae Veterinariae, v.47, n.1657, 2019.

PARANÁ. Secretaria de Estado da Agricultura e do Abastecimento. Caprinocultura Mundo - Brasil e Paraná. 15p. Curitiba, 2003. Disponível em: < www.agricultura.pr.gov.br/ arquivos/File/deral/caprinos_AGO_03.pdf > . Acesso em: 08 jun. 2020.

PARANÁ. Secretaria de Estado da Agricultura e do Abastecimento. A ovinocultura do Paraná no contexto nacional e mundial: um breve diagnóstico situacional. 15p. Curitiba, 2004. Disponível em: < www.agricultura.pr.gov.br/arquivos/File/PDF/ ovinosdiagset121103.pdf>. Acesso em: 8 jun.2020.

PEREIRA, P.F.V.; ROMAO, F. T. N. M. A.; PENZETI, E.; SANCHES, J. F. Z.; CURTI, J. M.; FLAIBAN, K. K. M. C.; LISBÔA, J.A.N. Importância da transfaunação no tratamento da acidose láctica ruminal aguda induzida em cabras e ovelhas. Pesquisa Veterinaria Brasileira, v. 38, p. 670-678, 2018.

PORTELA, R.A; RIET-CORREIA, F; GARINO JÚNIOR, F; DANTAS, A.F.M; SIMÕES, S.V.D; SILVA, S.M.S. Doenças da cavidade nasal em ruminantes no Brasil. Pesquisa Veterinária Brasileira. v.30, n.10, p.844-854, out 2010.

PUGH, D.G.; BAIRD, A.N.; EDMONDSON, M.A.; PASSLER, T. Sheep, Goat and Cervid Medicine. 3 ed. Filadélfia: Elsevier Inc, 2020. 576p.

SILVA, N.S; SILVEIRA, J.A.S; CAMPOS, K.F; SOUZA, M.G.S; LOPES, C.T.A; OLIVEIRA C.M.C; DUARTE, M.D; BARBOSA, J.D. Acidose ruminal em ovinos, diagnosticada pela Central de Diagnóstico Veterinário (Cedivet) da Universidade Federal do Pará, no período de 2000 a 2008. Ciência Animal Brasileira, Suplemento 1, p.191-196, 2009.

SILVA, T.V.; SANTOS, J.F.; SILVA, R.J.; CAJUEIRO, J.F.P.; SOUZA, J.C.A.; MENDONÇA, C.L.; AFONSO, J.A.B.; SOUTO, R.J.C. Achados clínicos e laboratoriais em bezerros da raça Gir leiteira com "Síndrome do bebedor ruminal". Ciência Veterinária nos Trópicos, v.19, n.1, p-58-64, 2016.

SUDRÉ, A.P.; LELES D.; LIMA M.F.; BOMFIM, T.C.B. First molecular characterisation of Giardia duodenalis infection in dairy goats in Brazil. Veterinarni Medicina, v.59, n.6, p.283-292, 2014.

SMITH, B. P.; VAN METRE, D.; PUSTERLA, N. Large Animal Internal Medicine. 6 ed. Missouri: Mosby, 2019. 1949p.

VIEIRA, A.C.S; AFONSO, J.A.B; MENDONÇA, C.L; COSTA, N.A; SOUZA, M.I. 2006. Estudo retrospectivo da acidose láctica em caprinos e ovinos atendidos na Clínica de Bovinos, Campos Garanhuns/ UFRPE. Revista Brasileira Ciências Agrárias. v.1, n.1. p.97-101, 2006.

XIMENES, L.J.F; CUNHA, A.M. Setor de peles e de couros de caprinos e de ovinos no Nordeste. Informe rural ETENE. v.6, n.1, 22p. mar, 2012. 